\title{
CONDICIONES, VOCES Y PERCEPCIONES DE JÓVENES DE LA UPN, DURANTE LA PANDEMIA POR COVID-19
}

\author{
CONDITIONS, VOICES AND PERCEPTIONS \\ OF YOUNG PEOPLE AT THE UPN, DURING \\ THE COVID-19 PANDEMIC
}

doi: 10.21555/rpp.v33i33.2407

\author{
Diego Rodríguez Calderón de la Barca \\ Universidad Pedagógica Nacional, México \\ diegorcb.upn@gmail.com \\ https:// orcid.org/0000-0003-4121-6396
}

Recibido: octubre 8, 2021 - Aceptado: noviembre 9, 2021

\section{RESUMEN}

El objetivo de este trabajo está orientado a conocer y describir las condiciones y desigualdades sociotecnológicas, así como algunas experiencias educativas y emocionales de las y los jóvenes de la Universidad Pedagógica Nacional Unidad 153, Ecatepec, durante la pandemia de COVID-19. El análisis parte de una perspectiva constructivista estructuralista de Pierre Bourdieu y de una metodología mixta, a partir de una muestra no probabilística por conveniencia y entrevistas semiestructuradas. Se analizaron las implicaciones de estudiar la carrera de manera no presencial, las condiciones materiales y las percepciones de las y los estudiantes durante el confinamiento. Los resultados muestran que un amplio porcentaje de los encuestados ha pensado en abandonar sus estudios, dado que la modalidad a distancia se ha empalmado con otras actividades como las domésticas y el cuidado de familiares, así como la falta de capitales tecnológicos, de ingresos y la imperante necesidad de trabajar, asumiendo distintos roles, además del de estudiantes. Por otra parte, el texto destaca que estudiar en casa influyó en ampliar las brechas de desigualdad al no contar todos con las mismas herramientas y servicios; no obstante, detonó otros aprendizajes relacionados con el uso de plataformas educativas e incidió en construir una mayor autonomía en el aprendizaje. Por lo tanto, este trabajo expone las distintas dificultades, desigualdades, percepciones y afectaciones emocionales a las que se enfrentó esta comunidad estudiantil, pero también resalta el ejercicio de su autonomía en torno a otros aprendizajes que coadyuban a su formación profesional.

Palabras clave: universidad, desigualdad social, pandemia, educación a distancia. 


\begin{abstract}
The objective of this work is aimed at knowing and describing the social and technological conditions and inequalities, as well as some of the educational and emotional experiences of the young people of the Universidad Pedagógica Nacional Unidad 153, Ecatepec, during the COVID-19 pandemic. The analysis starts from a structuralist constructivist perspective of Pierre Bourdieu and a mixed methodology based on a non-probabilistic sample for convenience and semi-structured interviews. The implications of studying the degree in a non-face-to-face way, the material conditions and the perceptions of the students during confinement were analyzed. The results show that a large percentage of those surveyed have thought about abandoning their studies, given that the distance modality has been linked to other activities such as domestic activities and caring for relatives, as well as the lack of technological capital, income and the prevailing need to work, assuming different roles, in addition to that of students. On the other hand, the text highlights that studying at home had an influence on widening the inequality gaps as not everyone had the same tools and services; however, it triggered other learnings related to the use of educational platforms and contributed to building greater autonomy in learning. Therefore, this work exposes the different difficulties, inequalities, perceptions and emotional affectations that this student community faced, but also highlights the exercise of their autonomy around other learning that contributes to their professional training.
\end{abstract}

Keywords: University, Social Inequality, Pandemic, Distance Education.

\title{
INTRODUCCIÓN
}

El presente artículo y la investigación que lo constituye, surgen por dos razones principales; la primera, debido a un interés propio por conocer más sobre la población juvenil de la Universidad Pedagógica Nacional 153 (Ecatepec), el lugar de trabajo en el que me desempeño como docente de tiempo completo; la segunda, visibilizar las condiciones y desigualdades sociotecnológicas a las que se han enfrentado las jóvenes de esta institución, especialmente durante la pandemia por el COVID-19 que inició a principios de 2020, generada por el virus SARS-Cov-2. La redacción está enfocada al género femenino, considerando que la matrícula universitaria de la UPN 153 está constituida por un 78\% de mujeres. En este sentido se hace uso principalmente del artículo «las» para referirnos a la población estudiantil que se analiza en este estudio.

La UPN 153, Ecatepec, es una universidad pequeña en tamaño y número de matrícula, ubicada en uno de los municipios más poblados e inseguros del Estado de México; empero, es una de las unidades en el municipio con mayor demanda estudiantil por la oferta educativa que ofrece actualmente: Licenciatura en Pedagogía, Maestría en Educación Básica, y Maestría en Enseñanza de las Humanidades (Historia, Lengua y Literatura).

El texto está dividido en cuatro apartados: en el primero, explico la metodología de diseño mixto que sustenta al estudio y el papel de los instrumentos de recolección de datos; en el segundo, destaco algunos aspectos centrales en torno a las desigualdades tecnológicas en México; en el tercer apartado, presento el contexto y la población de estudio, aunado al análisis sobre las condiciones, desigualdades y percepciones de las estudiantes de la UPN 153 a partir de la pandemia, y finalmente, expongo algunas consideraciones obtenidas de los resultados. 


\section{METODOLOGÍA}

El estudio lo desarrollé con base en una metodología mixta, utilizando una muestra no probabilística por conveniencia, constituida por 305 estudiantes. El criterio de inclusión fue: ser estudiante matriculada e inscrita en alguno de los semestres de la Licenciatura en Pedagogía de la UPN Unidad 153, o inscritas en algún posgrado de esta misma unidad. La encuesta la contestaron 300 estudiantes de licenciatura (de 19 a 24 años edad) y cinco de posgrado (de 25 o más años de edad), siendo un total de 305 universitarias, lo que representa un $84.5 \%$ de la población estudiantil correspondiente al ciclo escolar 2020-2021. Con base en el último corte del ciclo escolar antes mencionado, la población total universitaria está conformada por 362 estudiantes (337 de la Licenciatura en Pedagogía, 16 de la Maestría en Educación Básica, y nueve de la Especialización y Maestría en Enseñanza de las Humanidades).

Decidí utilizar metodología mixta en razón de que la población universitaria de la UPN 153 es pequeña con respecto de otras unidades de la UPN y de otras universidades, aprovechando así dos ventajas: 1) utilizar una muestra para las encuestas, conformada por la mayoría de la población estudiantil, y 2) complementar el análisis a partir de los dos instrumentos utilizados (encuestas y entrevistas) para contribuir, a partir de una análisis descriptivo, al conocimiento de la temática estudiada desde ambos enfoques (cuantitativo y cualitativo), no delegando todo a cifras o porcentajes ni tampoco a fragmentos de entrevistas, desarrollando un análisis más completo y detallado del estudio. Como bien explican Hernández, Fernández-Collado y Baptista: «la naturaleza compleja de la gran mayoría de los fenómenos o problemas de investigación abordados en las distintas ciencias están constituidos por dos realidades, una objetiva y la otra subjetiva» (2006, pp. 536-537).

La encuesta del estudio estuvo constituida por 41 preguntas, enviada a los correos de las estudiantes y contestada a través del formulario de Google. El levantamiento de datos se complementó con 12 entrevistas semiestructuradas a estudiantes de distintos semestres (segundo, cuarto, sexto y octavo) de la Licenciatura en Pedagogía, aprovechando así la simultaneidad que conlleva el diseño mixto en una investigación.

\section{Gráfica 1}

\section{Estudiantes de la licenciatura en pedagogía y posgrado}

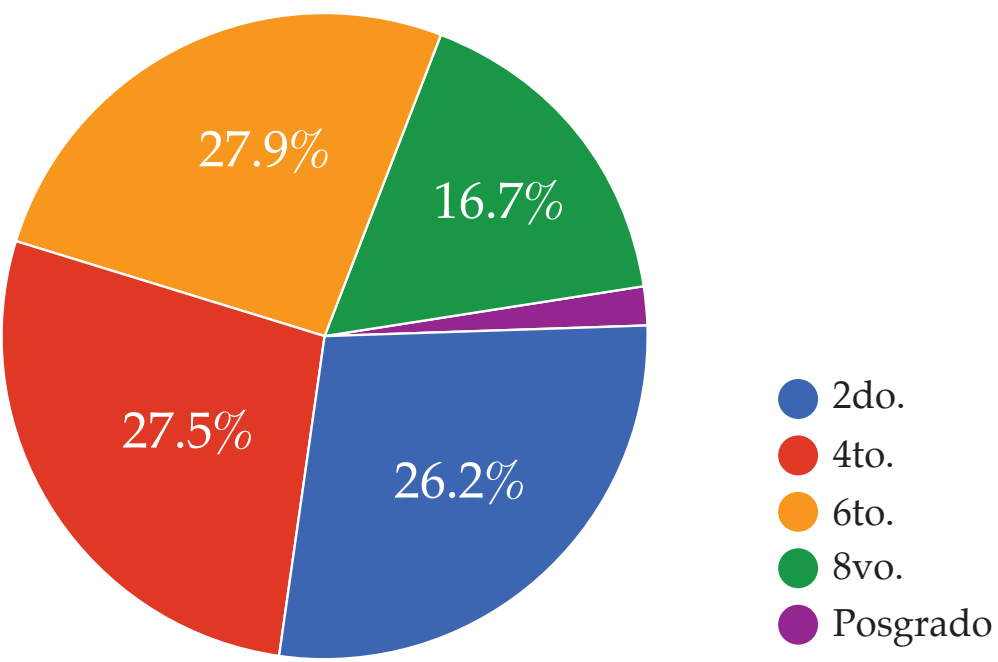


Para los fines de este artículo, me enfocaré solo a la interpretación de aquellas que se apeguen a los objetivos del texto, presentando porcentajes y gráficos correspondientes. Cabe señalar que las dimensiones abordadas en el estudio fueron: 1) contexto y situación familiar; 2) universidad y estudios; 3 ) aprendizajes en el contexto de pandemia; 4) papel de los docentes; 5) accesibilidad y equipamiento, y 6) bienestar físico y emocional.

Tanto las encuestas como las entrevistas se aplicaron, de manera simultánea, de febrero a junio de 2021. Entre las preguntas centrales, abordadas en el cuestionario y entrevistas, se encuentran las siguientes: ¿La pandemia afectó tu economía familiar o individual? ¿Cuáles son las principales dificultades a las que te has enfrentado, a partir de que empezó la pandemia y se interrumpieron las clases presenciales? ¿Cómo percibes o aprecias tu aprendizaje en la modalidad no presencial? ¿Qué dispositivo utilizas más para conectarte a tus clases? ¿La pandemia influyó para que actualmente trabajes de manera remunerada? ¿Consideras que ha incrementado tu autonomía en torno a tus aprendizajes en los últimos dos semestres? ¿Has pensado en interrumpir tus estudios en los últimos dos semestres? ¿Cómo consideras tu bienestar emocional actualmente?

A partir de la aplicación de estos instrumentos, y de una metodología cuantitativa y cualitativa, construí el análisis sobre las desigualdades y las diferencias entre las estudiantes en torno de sus condiciones y situaciones de accesibilidad, equipamiento y, por ende, capitales tecnológicos, así como otros aspectos relacionados con su formación, ocupaciones e implicaciones emocionales.

\section{DESIGUALDADES TECNOLÓGICAS DE LOS SISTEMAS EDUCATIVOS EN MÉXICO}

La población juvenil universitaria se caracteriza por ser heterogénea en distintos aspectos, entre ellos, las diferencias que existen en cuanto a las condiciones y desigualdades económicas, carencias relacionadas con su origen familiar y, particularmente, diferencias formativas; es decir, de jóvenes que asisten a universidades de prestigio, y por ende privilegiadas, frente a quienes acuden a universidades públicas con considerables carencias materiales y de equipamiento. Sin embargo, cabe señalar que, en términos generales, pero no de manera similar, la pandemia ha afectado a un gran número de estudiantes en todos los contextos.

\footnotetext{
En casi todo el mundo, una gran cantidad de estudiantes se han visto afectados de distintas maneras. La cancelación de clases presenciales y los estudios en línea, organizados con premura y preparación insuficiente, han generado grandes dificultades y se han incrementado los procesos de exclusión y marginación [...] En México, se recrudecen los problemas financieros, en particular para las universidades públicas en situación de crisis y con necesidad de recursos adicionales para su rescate económico y viabilidad (Ordorika, 2020, pp. 3-4).
}

Ahora bien, ¿los sistemas educativos en México estaban preparados para afrontar una crisis de salud como la que padecemos? La respuesta es claramente «no» y mucho menos el grueso de las familias a nivel nacional. La gran mayoría de las instituciones públicas educativas no cuentan actualmente con equipamiento, infraestructura y, mucho menos, con dispositivos que pueda utilizar la población estudiantil de todos los niveles educativos, para darle continuidad a sus estudios en una modalidad en línea. Actualmente, de acuerdo con Rieble y Viteri (2020), México cuenta parcialmente con conectividad en las escuelas; plataformas digitales, tutoría virtual y de paquete de recursos digitales. Únicamente, en donde existe un mayor recurso establecido es en los repositorios de contenido digital. 
Muchos son los problemas que han afectado al ámbito educativo en sus distintos niveles, que anteceden a la pandemia, pero esta los ha agudizado, sobre todo en las familias y jóvenes más vulnerables.

En México, la crisis educativa se percibe desde mucho antes de la pandemia: exclusión educativa, rezago, abandono y bajos niveles de aprovechamiento son, cuando menos, el diario acontecer dentro de las aulas de las instituciones públicas del país, mientras que, por otro lado, se encuentran la apatía docente, la fatiga laboral, el descontento general y la sobrecarga de funciones administrativas. Las medidas de aislamiento social han sacado de nuevo a la luz estos problemas, pero también han hecho visibles otros nuevos: la escasa cultura en TIC de la población escolar, las carencias en conectividad y disponibilidad de tecnología aplicable a la educación, así como la lentitud con la que las autoridades educativas han implementado las políticas y reformas educativas y, sobre todo, lo referente a la educación digital, necesaria para ciudadanía global. (Navarrete, Manzanilla y Ocaña, 2020, p. 164).

Así, la pandemia por COVID-19 ha dejado ver lo frágil que pueden ser los sistemas educativos en todos los niveles escolares a nivel global. De acuerdo con el Banco Interamericano de Desarrollo (BID) (2020), México es el segundo país con mayor interrupción escolar en niños y jóvenes de 6 a 17 años. El 16\% de los y las estudiantes que dejen la escuela, lo hará por motivos económicos y el $9 \%$ lo hará por razones académicas. Cifras semejantes se presentan en la Encuesta para la Medición del Impacto COVID-19 en la Educación (ECOVID-ED, 2020). Exclusivamente hablando de jóvenes que estudian una carrera universitaria, Roberto Rodríguez (2021), con base en la encuesta antes mencionada, explica que de los 89,940 estudiantes de educación superior que suspendieron sus estudios en el ciclo escolar 2019-2020, el $44.6 \%$ declaró que la pandemia por COVID-19 fue la razón principal para hacerlo.

Los retos que afrontan los sistemas educativos en México son bastantes y las carencias de los mismos repercutirán a corto y mediano plazo en la población estudiantil, afectando e influyendo en su desempeño, rezago educativo y en la interrupción escolar. Aunque estas problemáticas refieran más a un conflicto multifactorial, en el último año, la pandemia ha sido un factor preponderante que las ha agravado considerablemente.

Al respecto, desde hace poco más de un año, la publicación de trabajos académicos en torno al impacto de la pandemia en el campo educativo, ha sido constante y numerosa. En su mayoría, dichas publicaciones demuestran lo compleja que ha sido la situación. La Comisión Nacional para la Mejora Continua de la Educación (2020), en su texto Experiencias de las comunidades educativas durante la contingencia sanitaria por COVID-19. Educación media superior, presenta distintas experiencias referentes a la educación a distancia; en este texto se exponen las condiciones y dificultades a las que se han enfrentado los estudiantes de nivel básico y medio superior, relativas a la forma en la que toman sus clases y los inconvenientes, retos y las dificultades que afrontan los docentes cuando las imparten; como por ejemplo, la atención a estudiantes en condiciones de vulnerabilidad o la dificultad que implica el seguimiento y retroalimentación de muchos de ellos. En síntesis, el texto muestra la necesidad de atender enfáticamente ciertas deficiencias dentro del sistema educativo, agudizadas por la pandemia. Especialmente, en el nivel medio superior, destaca la atención a la cobertura, el seguimiento a estudiantes, el refuerzo a los aprendizajes y el rezago educativo, entre otras actividades orientadas a prevenir el abandono escolar.

El cambio radical vivido en el escenario educativo ante tales circunstancias, ha hecho visibles distintas necesidades, muchas de ellas novedosas; otras han mostrado una estrecha asociación con las carencias propias del sistema educativo, de sus actores principales y de los planteles; algunas tienen que ver con la disponibilidad de recursos, las condiciones de la infraestructura física, los equipamientos tecnológicos básicos y materiales educativos, además de las capacidades cognitivas y emocionales de los actores (MEJOREDU, 2020, p. 7). 
Es importante mencionar que la educación a distancia no es exactamente una modalidad nueva. Algunos antecedentes de esta tienen que ver con distintas políticas emitidas desde mediados de siglo XIX y que poco a poco se han ido ampliando, modificando y cambiando de acuerdo con las condiciones sociales y contextuales. Algunos ejemplos de instituciones que comenzaron trabajando bajo esta modalidad son:

Escuela de Radio de Difusión Primaria para Adultos, en 1941; el Instituto Federal de Capacitación del Magisterio, en 1947; el Centro de Educación Básica de Adultos y Telesecundaria, en 1968; el Centro para el Estudio de Medios y Procedimientos Avanzados de la Educación, en 1971; el Sistema de Universidad Abierta de la Universidad Nacional Autónoma de México, en 1972; la aplicación de un modelo de Preparatoria Abierta, en 1973; el Sistema Abierto de Enseñanza del Instituto Politécnico Nacional, en 1974 (Navarrete y Manzanilla, 2017, pp. 78-79).

Posteriormente, como afirman Navarrete, Manzanilla y Ocaña (2020), a finales del siglo XX y principalmente durante el siglo XXI, se han instaurado departamentos enfocados a la Educación a Distancia; uno de los más representativos es el Sistema Universidad Abierta y Educación a Distancia (SUAyED), de la UNAM.

Si bien es cierto, la identidad y la condición de joven universitario se relaciona con el espacio, el contexto y la experiencia presencial, en la actualidad observamos que la identidad de los y las jóvenes también se logra a partir de su adscripción institucional, conectándose a sus clases en línea y sintiéndose parte de una institución universitaria, sin necesidad de estarlo de manera física en ella.

Dentro de las diversas maneras de vivir la condición estudiantil, la presencia física en el espacio educativo ya no es una condición necesaria para ser o sentirse estudiante, prueba de ello son los sistemas abiertos y a distancia, que implican un tipo de vínculo más flexible con la institución, pero sobre todo con el conocimiento y el sentido que se le atribuye (Guzmán, 2013, p. 13).

Sobre el impacto de la pandemia en la educación superior, Miguel (2020) en su publicación La educación superior en tiempos de pandemia: una visión desde dentro del proceso formativo, ofrece un análisis sobre el sentir de los estudiantes, docentes y algunos administrativos de tres universidades del Valle de Oaxaca, ante el cambio de trabajo de una modalidad presencial a una a distancia. Dos de las universidades que se analizaron fueron privadas, una con infraestructura y reconocimiento alto, y otra con menor infraestructura y menor reconocimiento; la tercera universidad estudiada fue pública, con amplia infraestructura y reconocimiento social. Lo interesante es que, sin importar el tipo de universidad, la gran mayoría de los estudiantes mencionaron estar inconformes, estresados y con obstáculos recurrentes, como la mala comunicación y la falta de acceso a internet, que obstaculizaban su aprendizaje.

Como afirma Miguel (2020), más que un cambio de modalidad, fue un «ajuste emergente» en donde las tecnologías de la información y la comunicación continúan teniendo un papel central en la formación de los estudiantes. Sin embargo, como explica el autor en su texto:

Se observa una disparidad entre los avances tecnológicos y las competencias para aprenderlas y enseñarlas, es decir, las competencias para la sociedad del conocimiento, puesto que, tanto estudiantes como docentes, manifiestan requerir apoyo y ver como obstáculo la nueva modalidad emergente de tomar clases de manera virtual. Esto quiere decir que la edad o generación no es un determinante para saber más o menos sobre las tecnologías; esta realidad muestra lo que los eruditos de las tecnologías del aprendizaje y el conocimiento (TAC) han subrayado, que la educación actual está en crisis debido a que existe un desajuste entre los avances tecnológicos, los currícula, las metodologías y las necesidades de los estudiantes (Miguel, 2020, p. 37). 
Por otra parte, Antezana, Czarny y Salinas (2021) presentan, a partir de metodología cualitativa, las condiciones y valoraciones (sentidos y significados) del ser universitario, especialmente de estudiantes de la Licenciatura en Educación Indígena de la UPN Ajusco. En este estudio se profundiza en cómo la pandemia afectó a los estudiantes en los aspectos socioeconómicos y tecnológicos. Un dato interesante es que, al empezar a estudiar fuera de la universidad, los estudiantes se vieron afectados por necesitar de los recursos que su universidad les ofrece y que, entre los más valorados por los jóvenes de la LEI, están: el comedor, los equipos de cómputo, la red inalámbrica y la biblioteca; recursos imprescindibles para su formación. Por lo mismo, cuando inició la modalidad de trabajo a distancia, para muchos, la situación resultó ser más complicada de lo esperado, influyendo y determinando en que regresaran a su comunidad de origen (Chiapas, Oaxaca, Puebla, entre otros estados). En palabras de las autoras: «algunos estudiantes, los menos, abandonaron sus estudios o reprobaron materias. Esto demostró cómo afectan a la continuidad de los estudios las brechas económicas y tecnológicas» (Antezana, Czarny y Salinas, 2021, p. 78).

Hasta aquí, lo presentado da cuenta de algunos de los textos que reflexionan y analizan los principales retos y las dificultades a los que nos enfrentamos en México durante la pandemia. Y, aunque buen número de personas en México y en el mundo ya están vacunadas, los contagios continúan (incluyendo algunos por las nuevas variantes del virus), por lo que resulta trascendente trabajar con y desde las distintas instituciones de gobierno, y con las instituciones educativas en todos los niveles escolares, e involucrar a todos los actores sociales posibles para afrontar, de la mejor manera, la contingencia en la que vivimos actualmente.

\section{UN ACERCAMIENTO A LAS CONDICIONES, VOCES Y PERCEPCIONES DE ESTUDIANTES DE LA UPN 153, DURANTE LA PANDEMIA POR COVID-1}

En este apartado presento el análisis realizado a la comunidad universitaria estudiada. En primera instancia, se puede constatar que, con base en la información y datos obtenidos en los cuestionarios y entrevistas, la gran mayoría de las estudiantes de la UPN 153, de acuerdo con Bourdieu y Passeron (2003), son pioneras y forman estudiantes de primera generación que ingresan a la universidad; es decir, no cuentan con una tradición escolar familiar en estudios superiores; únicamente el $13.1 \%$ de las y los estudiantes encuestados tiene padre con educación superior y $8.9 \%$ de las jóvenes tiene madre universitaria. La gran mayoría de padres y madres estudiaron la secundaria con el $38.4 \%$ y $39.4 \%$ respectivamente, seguido por el bachillerato (20.3\% padres y $15.4 \%$ madres), la primaria (entre el $14.4 \%$ padres y $15.4 \%$ madres) y el estudio de una carrera técnica (entre $7.5 \%$ padres y $13.5 \%$ madres). Por lo tanto, si bien el estudio se enfocó en una misma comunidad universitaria, las estudiantes, su familia de origen, sus recursos y condiciones en su hogar, son distintas.

Aunque todos los sujetos que ingresan a una institución pasan a formar parte de eso que se llama comunidad universitaria y cuentan con un número de matrícula que los identifica como estudiantes, es imprescindible conocer quiénes son, en qué se parecen y en qué son disímiles (De Garay, Miller, y Montoya, 2016, p. 98).

En relación con lo anterior, distintos autores (Bourdieu y Passeron, 2003; Silva, 2013; Blanco, Solís, Robles, 2014), demuestran que el nivel escolar de un familiar y los capitales de la familia de origen influyen, e incluso llegan a determinar, la trayectoria y el desempeño escolar de las estudiantes. Esto representa una primera desigualdad educativa intra e interuniversitaria, pues el origen familiar tiene un impacto considerable en la trayectoria de las estudiantes y más aún, cuando los capitales tecnológicos y recursos económicos están teniendo 
un papel trascendental en la continuidad y formación escolar y profesional, de los niños y jóvenes; aún más, cuando para algunos, el apoyo y uso de recursos depende solo de los que ofrece la universidad (equipos de cómputo, internet, un espacio para estudiar, etcétera).

Es cierto que los campos en los que se desenvuelven los estudiantes y su disposición de los capitales (tecnológicos, como el internet y la accesibilidad), influyen en su desempeño estudiantil y profesional; sin embargo, también es cierto que, de acuerdo con la propuesta de Giddens (1995), en torno de su teoría de la estructuración, la acción y capacidad de agencia, los agenciamientos juveniles a través las decisiones y la forma en que afrontan sus situaciones desde su condición de estudiantes, cobran relevancia en su desenvolvimiento universitario, profesional y laboral, así como la manera de hacer frente a los efectos de la pandemia.

Las diferencias entre estudiantes pueden ser abismales, y más entre aquellos que tienen mayores carencias en términos materiales y de servicios. Esto representa una desigualdad más, entre quienes cuentan con el apoyo familiar para solventar sus gastos universitarios y quienes lo tienen que hacer por su propia cuenta. Con base en lo anterior $-64 \%$ de la población encuestada-, los padres les pagan sus estudios; no obstante, un porcentaje considerable (30.5\% de las jóvenes) se hacen cargo por sí mismas de los gastos que implican sus estudios universitarios.

\section{Gráfica 2 \\ Estudiantes con respecto a la persona que paga sus estudios}
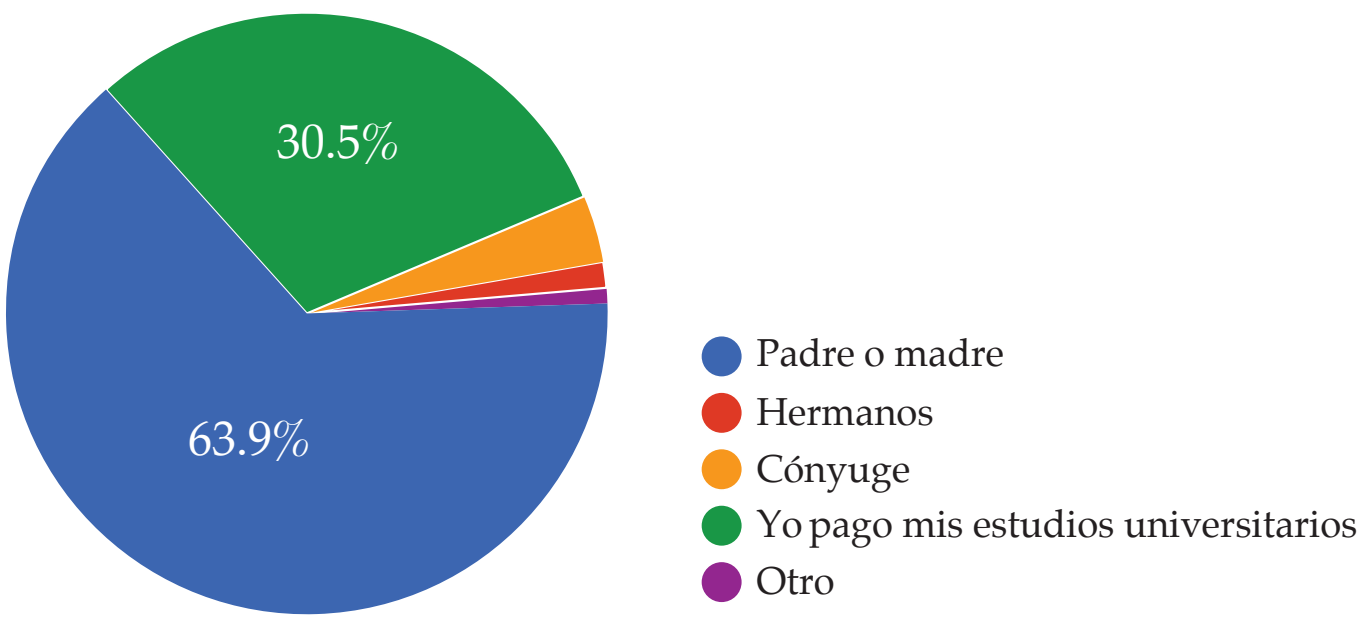

Por lo tanto, el papel de las becas puede ser tan significativo a tal grado que constituye un apoyo determinante para que las y los estudiantes interrumpan o no sus estudios. Los casos de estudiantes que cuentan con una beca en la UPN 153, con base en la muestra de este estudio, es solo del 14\%; un porcentaje menor al de años anteriores. Un problema ocasionado directamente, y en gran medida, por el fuerte recorte federal a partir del gobierno en turno.

Los resultados del estudio también constatan que la pandemia y sus consecuencias (contagios y en el peor de los casos, pérdidas familiares) trastocó directamente las interacciones, emociones y expresiones afectivas de los estudiantes hacia sus familiares y amistades, generando un extrañamiento importante a ciertas prácticas y afectividades entre los miembros de 
sus familias, así como entre sus compañeros. Al respecto, existen diversos estudios que analizan puntualmente este tema (González, 2020; Miguel, 2020; Navia, Czarny y Salinas, 2021).

Por otro lado, la pandemia modificó directamente las rutinas de las y los jóvenes, por lo que empezaron a asumir nuevas responsabilidades; el $87.5 \%$ de las jóvenes tuvieron que dedicar tiempo considerable a las labores domésticas y al cuidado de familiares como abuelos, padres, sobrinos o hermanos. No obstante, las nuevas responsabilidades no se acotaron únicamente al contexto familiar o al hogar, sino que trascendieron al mercado laboral, generando una reorganización tanto dentro como fuera de casa. Algunos de los estudiantes encuestados salieron junto con sus familias a otros estados para residir de manera temporal e indefinida, aprovechando el trabajo y el estudio a distancia. En este aspecto, es visible la diferencia de género en torno a dichas actividades, las mujeres son quienes más trabajan dentro de casa, sumado a actividades universitarias. Cabe resaltar que la gran mayoría las personas entrevistadas y de quienes contestaron la encuesta, fueron mujeres, lo que inclina los resultados hacia el género femenino.

Casi el $70 \%$ de las estudiantes afirmó que la pandemia afectó su economía familiar o individual, por lo que un porcentaje considerable de las jóvenes debieron empezar a trabajar temporal o indefinidamente, para contribuir a los ingresos familiares o para contar con ingresos propios.

\section{Gráfica 3}

\section{Estudiantes quienes consideran que la pandemia afectó su economía familiar y/o individual}
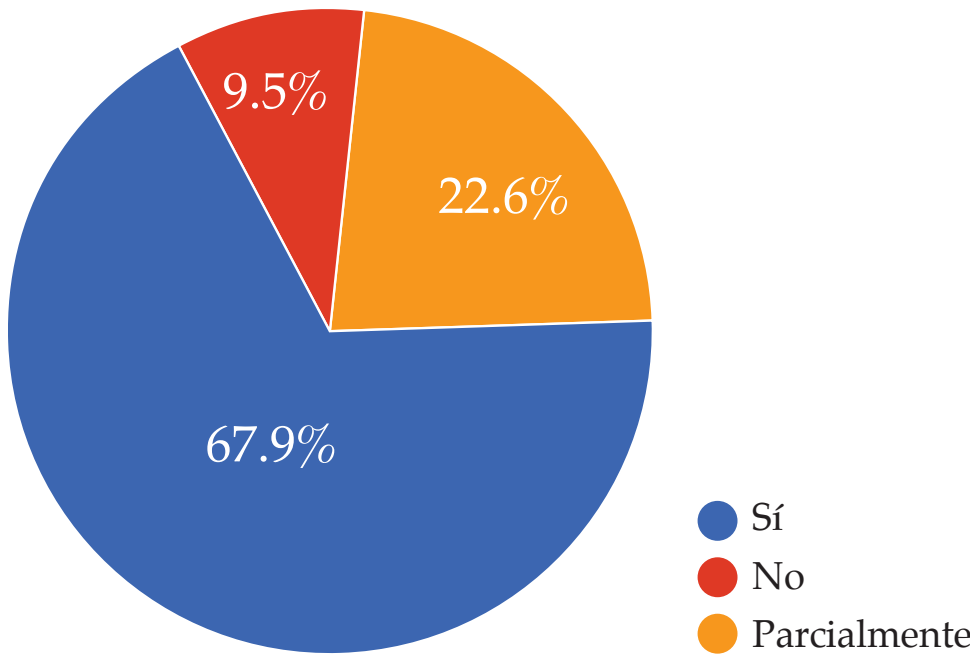

En relación con lo anterior, el riesgo de que las jóvenes universitarias de esta universidad interrumpan sus estudios está latente por el impacto de la pandemia. Para la gran mayoría de las familias no ha sido fácil sobrellevar su situación económica; un porcentaje bajo de estudiantes cuenta con beca, mientras que otros empezaron a trabajar a partir de la pandemia; existe una clara correlación de sus respuestas, cuando se les preguntó si actualmente trabajan de manera remunerada; el 35.7\% contestó que sí, mientras que el 33.4\% respondió que la pandemia influyó para que empezaran a trabajar. Como lo expresan a continuación:

- Sí ha habido un cambio, totalmente. Mi papá se dedica a la publicidad, entonces al no haber eventos por la pandemia, él no tiene trabajo. Tuvimos que cambiar radicalmente la rutina. En mayo había solvencia, pero ya en agosto de 2020, fue donde la empresa ya no aguantó y tuvieron 
que cerrar. Ahorita, por ejemplo, doy clases de inglés y tengo tres niñas a las que les ayudo a hacer sus tareas porque su mamá sale a trabajar. De hecho, es mi primer trabajo (Romina, estudiante de Pedagogía).

- Anímicamente nos afectó mucho, porque hubo un tiempo que nos dio COVID a todos y tuvimos que gastar mucho, [y] estar saliendo al médico. Hubo bastantes gastos, tuvimos que conseguir un tanque de oxígeno y muchas medicinas (Verónica, estudiante de Pedagogía).

El ingreso al trabajo por parte de las estudiantes no es visto como algo negativo, por el contrario, ellas mismas consideran que pueden ganar experiencia laboral, principalmente cuando esta está relacionada con su profesión o cuando son actividades que tienen que ver con la formación y la educación (por ejemplo, auxiliar a padres o madres de familia para el cuidado y apoyo en las tareas de sus hijos o hijas). Esto, además de contribuir a su formación, es un ingreso importante para sus gastos personales universitarios o para aportar a los gastos familiares.

- Yo ahorita doy clases de manera particular, presencial y en línea, así como nosotros tomamos clases. Doy tutorías a niños, desde nivel preescolar hasta nivel preparatoria; cobro por hora. Aunque esto lo vengo haciendo desde cuarto semestre, y de alguna manera para enriquecer mi práctica docente, lo que yo gano lo invierto en mi educación. A la semana tengo diez niños, tengo dos de prescolar, seis de primaria y dos de secundaria [...]. Es asesoría general, trabajo presencialmente con niños de prescolar con todas las medidas de prevención, porque con ellos hay que practicar motricidad fina, motricidad gruesa, números, etcétera (Andriana, estudiante de Pedagogía).

Un aspecto a resaltar acerca de los cambios que produjo la pandemia actual, es que, como se expone en párrafos anteriores, varios jóvenes empezaron a trabajar para apoyar en los gastos familiares, superponiendo el empleo actual a la universidad, agravándose así la incertidumbre y las dificultades para permanecer y concluir sus estudios superiores. Por lo anterior, uno de los hallazgos más llamativos, con un sentido negativo en el estudio, es el alto riesgo que existe de interrupción escolar: el 55\% de las estudiantes encuestadas confirmó que, en los dos últimos semestres, ha pensado en interrumpir sus estudios.

Otra de las problemáticas que se suma a las mencionadas es la de los efectos del encierro, especialmente en los aspectos emocionales, físicos y psicológicos presentes en las jóvenes encuestadas y en sus familiares. Mientras que algunas padecieron malestares emocionales por cuestiones o motivos económicos, otras se vieron afectadas, no solo por cuestiones económicas, sino también por las condiciones de salud.

Los porcentajes en torno al bienestar emocional de las estudiantes no son muy alentadores, teniendo en cuenta que estos se concentraron en quienes consideran tener un bienestar emocional deteriorado (43\%) o malo (17.4\%), en contraste con quienes respondieron que su bienestar es bueno (32.8\%) o excelente (6.5\%). En relación con el bienestar físico, casi la mitad de la población (48.9\%) respondió que su bienestar físico es bueno, pero 39\% contestó que está deteriorado; los menores porcentajes se acotaron a excelente $(5.6 \%)$ y malo $(6.6 \%)$. El $83.3 \%$ de las encuestadas contestaron que se consideran afectadas emocionalmente por el confinamiento: un factor preponderante de este malestar se debe a cuestiones de tensión relacionadas con la pérdida de algún familiar cercano.

- Ahorita estoy viviendo con la esposa de mi papá, porque mi papá acaba de fallecer y efectivamente la pandemia afectó la economía familiar (Alicia, estudiante de Pedagogía).

- A mi mamá también la despidieron, pero logró insertarse a otro trabajo. Se movieron muchas cosas. Hubo mucha ansiedad para mí y para mi mamá. Cuando ella enfermó, mi desempeño en 
la universidad bajó mucho, entonces hubo repercusiones en mí, pero poco a poco nos fuimos adaptando a la situación (Adriana, estudiante de Pedagogía).

Las afectaciones emocionales y económicas generaron, a su vez, un estrés y deterioro físico mayor al común, vivido durante las actividades universitarias cotidianas, experimentadas en el estudio de manera presencial.

- El estrés es mucho por la cuestión económica; tenemos que «buscarle» para salir, tenemos responsabilidades para salir, tengo mi trabajo y ese sí no lo puedo dejar. Y sí, hago un poco de ejercicio, pero paso mucho tiempo sentada para mis clases y las clases que tengo que dar, a veces me duele la espalda (Mariana, estudiante de Pedagogía).

- Pues más que malestar mental, he tenido problemas de circulación, así como dolor de cuello y espalda, porque pasamos mucho tiempo sentados frente a la computadora por las clases y los trabajos (Roxana, estudiante de Pedagogía).

Es importante destacar esta otra mirada sobre los efectos emocionales y físicos de los estudiantes que ha originado la pandemia, puesto que incide en su desempeño, en su motivación e incluso en su continuidad.

Por otro lado, sabemos que las desigualdades que padecen los jóvenes son multidimensionales; sin embargo, atendiendo los intereses del presente texto, las principales desigualdades sociotecnológicas identificadas en las jóvenes universitarias de la UPN 153, se relacionan con el acceso a internet y la insuficiencia de equipos de cómputo de uso personal. Hay jóvenes que se encuentran en desventaja de capitales culturales, educativos, económicos y tecnológicos. En cuanto a las principales carencias mencionadas por las estudiantes, están las siguientes: 1) falta de dispositivos para acceso a clases virtuales y elaboración de trabajos; 2) tiempo; 3) accesibilidad a internet; 4) dinero, y 5) luz.

\section{Gráfica 4 \\ Estudiantes con respecto a las principales carencias relacionadas a recursos para sus clases virtuales}

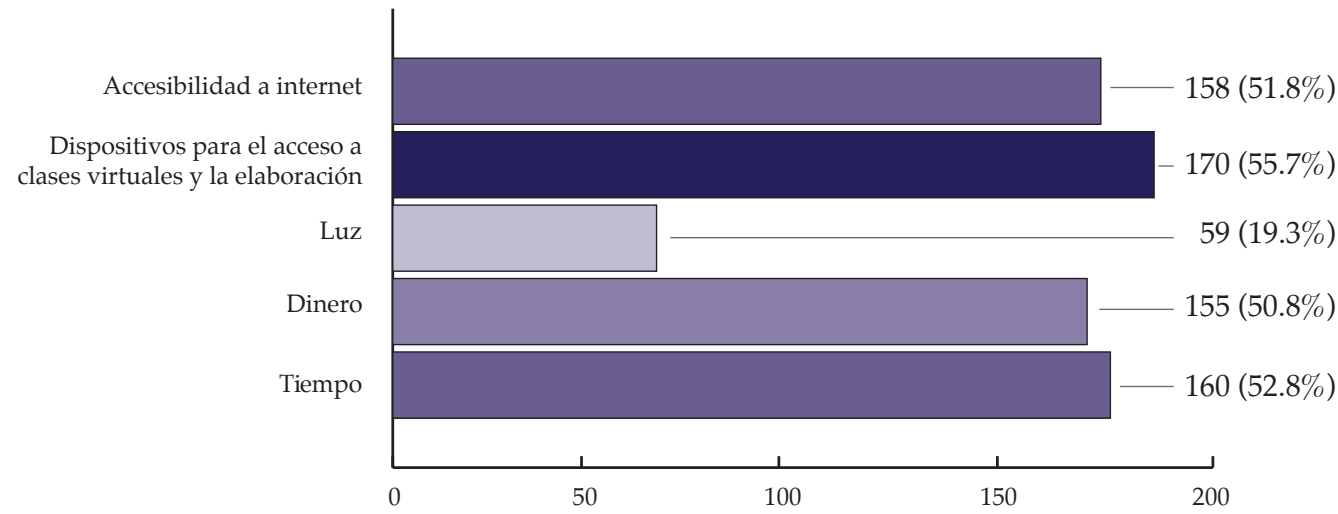

El dato que más llama la atención es que consideran que el tiempo es uno de los recursos que más está en disputa, pues cuando estudiaban en modalidad presencial estaban hasta más de seis horas en la universidad, mientras que ahora requieren pasar hasta más de 10 horas, dedicándolas al trabajo universitario. 


\section{Gráfica 5}

Estudiantes y número de horas que pasan frente a algún dispositivo para tomar clase y hacer tus tareas escolares

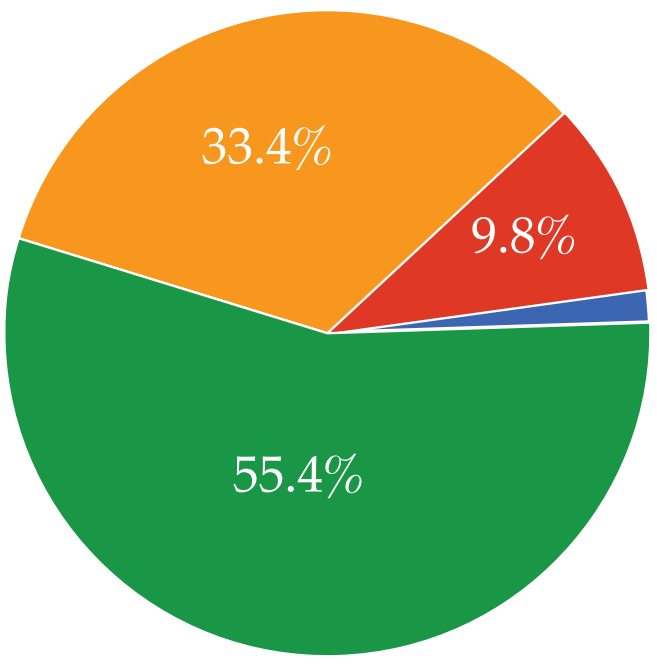

Uno de los datos quizá no sorprendentes, pero que sí abonan a entender por qué muchos jóvenes tienden a conectarse frecuente y preferentemente con su celular, es porque no tienen un equipo de cómputo propio, y si lo tienen, se ven en la necesidad de compartirlo, especialmente con sus hermanos $u$ otros familiares. Los resultados muestran que un porcentaje importante, el $54.4 \%$ de los estudiantes, comparte su equipo o laptop con familiares (principalmente con sus hermanos). El 26.9\% cuenta con equipo propio y es de uso personal (no tienen que prestarlo), y el 18.7\% no tiene equipo propio por lo que necesitan pedirlo prestado, lo cual contribuye a que aumente el estrés por la entrega de las actividades escolares y merme su desempeño por no tener una herramienta propia de trabajo.

La gran mayoría de estudiantes se conecta a sus clases con un equipo de cómputo (39.6\%) o teléfono celular (59.4\%); la generalidad de las veces son equipos básicos para ver y escuchar las clases, pero no para trabajar desde ellos, por la poca comodidad visual que ello conlleva a la hora de hacer tareas o trabajos. La practicidad del celular radica en que es el dispositivo con el que pueden conectarse con datos o wifi desde distintos lugares para, al menos, escuchar la clase sin ver ni ser vistos por sus docentes.

Dentro de la población universitaria estudiada, hay jóvenes más favorecidas que cuentan con los capitales necesarios o suficientes (equipamiento, servicio de internet, etcétera), y que durante las entrevistas expresaron ideas que demuestran lo cómodo y práctico que puede ser continuar en esta modalidad.

- La verdad yo estoy muy cómoda trabajando así, me da tiempo de hacer muchas más cosas y como tal no he tenido carencias, tengo mi laptop desde la secundaria y mi celular, entonces es cuestión de cuidarlo, ahora sí que antes de esto de la pandemia, ellos [su papá y mamá] ya habían comprado esas cosas para nosotras y esa laptop la uso de manera individual [...]. En cuanto al servicio de internet, como mi tía es mi vecina, ella nos proporciona su clave para conectarnos (Romina, estudiante de Pedagogía). 
Varias estudiantes expresaron ideas semejantes, relacionadas con las distintas actividades y experiencias que han debido integrar a su día a día, y que no se acotan únicamente a actividades universitarias. Estas ideas ayudan a tener un mejor panorama sobre lo que muchas de ellas vivieron durante el confinamiento y en las que expresan su preocupación por su situación educativa.

- Las horas frente a la computadora son muchas y están afectando mi vista tremendamente (Salma, estudiante de Pedagogía).

- Piensan que todos vivimos en condiciones dignas de estudio (Mariana, estudiante de Pedagogía). - Deberían tomar en cuenta las necesidades de los alumnos y el tiempo que pasamos frente a la computadora, que son muchas (Susana, estudiante de Pedagogía).

Por lo tanto, existen aspectos y situaciones que se conjugan y afectan más la condición de estudiantes, la falta de recursos, capitales tecnológicos y el tiempo que destinan a ocupaciones ajenas a las escolares, acumulando así desventajas en su desenvolvimiento estudiantil. De acuerdo con Ramírez (2013, p. 260), «se ha observado que los estudiantes de sectores desfavorecidos conciben expectativas que tienden a ser débiles, tímidas, incluso más que sus "posibilidades objetivas", de llevarlas a la práctica».

En cuanto al aprendizaje, hay datos y respuestas que contrastan sobre la manera de percibir su aprendizaje, el cual tiene mucho que ver con sus condiciones y capitales objetivados (dispositivos, internet, contar con algún espacio propio para estudiar, un cuarto con escritorio, etcétera), y el tiempo que cada uno dedica y disponga para estudiar. Como se observa en las siguientes gráficas, un porcentaje considerable de la población encuestada respondió que su aprendizaje ha decaído, lo que deja entrever que las clases en línea no han sido la mejor opción, pero que funcionan como una actividad paliativa para que no abandonen sus estudios.

\section{Gráfica 6}

\section{Estudiantes y su percepción sobre su aprendizaje a partir de clases en linea}

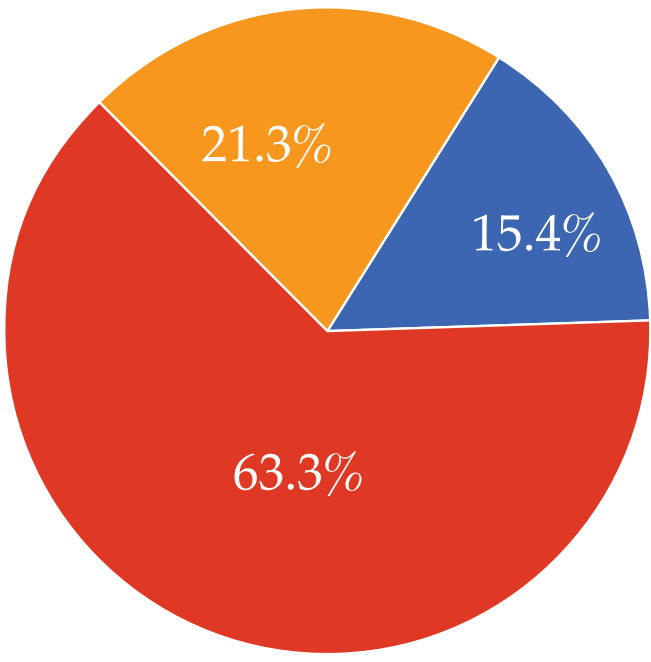

Sí, ha mejorado mi aprendizaje

Sí, ha decaído mi aprendizaje

No, ha sido el mismo 
En torno a la apreciación de su aprendizaje en los dos últimos semestres, poco más de la mitad de las respuestas se concentraron entre un aprendizaje elemental (39\%) y deficiente $(14.8 \%)$. Mientras que el $43.3 \%$ comentó que su aprendizaje había sido bueno y $2.9 \%$ excelente. Sin embargo, también existen testimonios que consideran que los aprendizajes han sido favorables.

¿Y cuál es tu apreciación del aprendizaje en esta modalidad?

- Pues yo creo que estoy aprendiendo bien, incluso creo que interactuamos más en nuestras clases por lo mismo que los profes piden nuestra opinión, cosa que no pasa en presencial, y ahorita sí nos da el tiempo para que hagamos el debate. Y así siento que aprendo más (Pamela, estudiante de Pedagogía)

- Creo que el aprendizaje es el mismo, depende del desempeño del alumno, si tú eras un alumno desatendido en clases presenciales, que no te importaba entrar o poner atención, pues obviamente harás lo mismo en clases a distancia. Pero si eres un alumno a quien realmente le importa aprender, poner atención, obviamente vas a estar al pendiente de los maestros, de las tareas. Entonces creo que sí influyen los factores externos, pero realmente si un alumno se empeña, lo va a lograr (Alicia, estudiante de Pedagogía).

Dichas respuestas expresan también las diferencias entre unos y otros estudiantes de acuerdo con las ventajas y desventajas de cursar la universidad de manera no presencial.

- Bueno, yo no tengo ningún problema en esta modalidad, pero sí he escuchado que algunos de mis compañeros se quejan e incluso ya quieren regresar. Pero yo digo: si te organizas y te enfocas en lo que estás haciendo, no tienes ningún problema en aprender, pero sí se distraen muy fácil porque hay factores de distracción, sean las clases presenciales o no (Romina, estudiante de Pedagogía).

Hay elementos a resaltar acerca del modo no presencial, como lo expone el informe sobre Los jóvenes y la COVID-19. Efectos en los empleos, la educación, los derechos y el bienestar mental (OIT, 2020), el impacto de la pandemia en las y los jóvenes ha sido sistemático, independientemente de que estudien, trabajen o estudien y trabajen. La trascendencia del cierre de escuelas y universidades, representa un impacto profundo en lo que es ser estudiante universitario; la socialización que se construye en el interior del aula y la propia universidad ha cambiado, por ende, la relación entre estudiantes, entre estudiantes y docentes, así como entre estudiantes y otros actores educativos.

No obstante, la universidad continúa siendo uno de los campos e instituciones trascendentes para la socialización y formación de los jóvenes, así como para la adquisición de habilidades y conocimientos orientados a su campo profesional y laboral.

Un aspecto a resaltar en relación con lo anterior es que, si bien el aprendizaje de las estudiantes se vio trastocado por las clases en línea, también sobresale que un grueso del porcentaje respondió que su autonomía en el aprendizaje incrementó, o incrementó parcialmente, en los dos últimos semestres. 


\section{Gráfica 7}

Estudiantes y el incremento de su autonomía en su aprendizaje en los dos últimos semestres

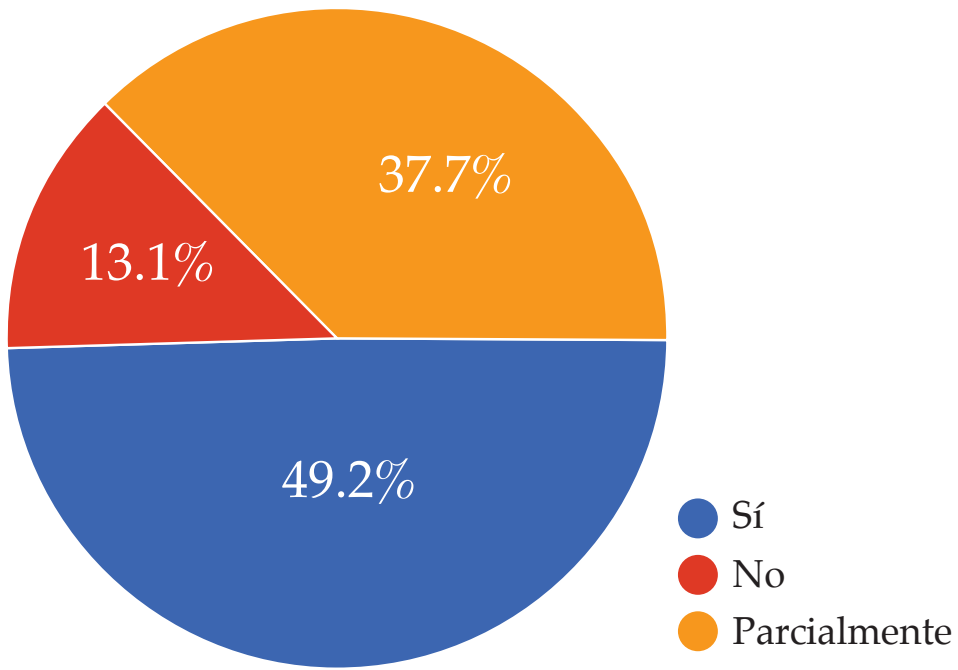

De este modo, su formación (necesaria) para su inserción al mercado laboral, ha adquirido nuevos parámetros, matices, conocimientos extracurriculares y por supuesto han desarrollado otras habilidades digitales, aprendiendo el uso de plataformas educativas como Classroom (de Google), Schoology, Moodle, entre otras. No obstante, cuando las condiciones en el hogar no son semejantes entre las estudiantes de un mismo contexto universitario, es cuando comienzan a generarse o reforzarse desventajas que pueden marcar una diferencia considerable en su aprendizaje, desempeño académico, evaluación por parte de los profesores y posible interrupción.

Los hallazgos de este estudio también apuntan a que las jóvenes encuestadas adquirieron nuevos y recientes aprendizajes, que quizá no tengan que ver del todo con el currículo institucional, pero que podrán utilizar como capitales educativos, además de desarrollar habilidades y aprendizajes relacionados con las medidas sanitarias, formas distintas de estudiar y socializar a distancia, emprender proyectos (comenzar un negocio o actividad remunerada, por ejemplo), modificar relaciones interpersonales, hábitos de cuidado de la salud, valorar lo que tienen, etcétera.

\section{CONCLUSIONES}

En el estudio se constata que, efectivamente, la pandemia influyó considerablemente para que los aprendizajes y la formación de las universitarias no fueran los mejores. Esto por varias razones: en primer lugar, los profesores, estudiantes y mucho menos las familias, no estábamos preparados para asumir un confinamiento de esta dimensión en el que, para muchos, se modificaron las ocupaciones profesionales y laborales; en segundo, es imprescindible reconocer la diversidad de condiciones familiares y del hogar que afrontaron las estudiantes y que pueden incidir en su desempeño escolar, incluso llegando a ser las responsables de interrumpir sus estudios; en tercero, es de suma importancia fortalecer las habilidades digitales o para la educación a distancia, tanto en estudiantes como profesores, pues estos constituyen 
capitales educativos en el campo escolar; en cuarto lugar, es fundamental identificar las fallas e insuficiencias en torno a los aprendizajes de los estudiantes, así como resaltar los recientes aprendizajes extracurriculares y significativos que se generaron a partir de la pandemia en el ámbito educativo.

Al respecto, la International Association of Universities (IAU, Marinoni, Van't Land y Jensen, 2020) menciona en su encuesta que la pandemia también representa una «oportunidad importante para proponer posibilidades de aprendizajes más flexibles, explorar aprendizajes híbridos o mezclados, y combinar aprendizajes sincrónicos y asincrónicos» (p. 11).

Si bien es cierto que la pandemia afectó en distintos ámbitos como el escolar, económico y emocional, también trajo consigo adecuaciones en la manera en que se desenvolvían en sus actividades cotidianas, sean jóvenes privilegiadas o no, pues todas modificaron su condición de universitarias y, en algunas, incluso favoreció para que construyeran una autonomía en torno al conocimiento y aprendizaje de plataformas digitales educativas, y para realizar otras actividades. Así, mientras algunas jóvenes han redoblado esfuerzos junto con sus padres, madres o familiares para continuar en la universidad, otras no lo han logrado o están en riesgo de interrumpir sus estudios, debido a que están asumiendo otras ocupaciones de manera paralela a sus estudios, como: trabajar, hacer labores domésticas u orientadas al apoyo y cuidado de familiares cercanos (hermanos, sobrinos, etcétera); en sí, responsabilidades que antes de la pandemia no tenían. Lo anterior dificulta su situación estudiantil y más cuando no cuentan con los recursos y las condiciones necesarias.

Como se mencionó, un 55\% de las estudiantes declaró haber pensado interrumpir sus estudios en el último año; es un porcentaje significativo pues, aunque entre las estudiantes encuestadas y entrevistadas no se identificaron casos concretos de jóvenes que estén decididas a interrumpir sus estudios, este es un problema vigente que debe ser considerado por el consejo universitario y las autoridades correspondientes, para tomar acciones y decisiones urgentes enfocadas a la prevención de la interrupción.

Entre algunas de las acciones preventivas para evitar la interrupción, pueden contemplarse conversatorios acerca de la importancia de concluir sus estudios; conocer las necesidades y solicitudes de los estudiantes, e implementar estrategias desde la institución, para incentivar la permanencia e identidad universitaria de las jóvenes. Flexibilizar el currículo o la malla curricular también de acuerdo con el plan de estudios, involucrando más aspectos y aprendizajes situados, relacionados a contextos reales y actuales que involucren el uso de plataformas digitales, temas relacionados a la pedagogía de la emociones, poner atención en la comprensión y aprendizajes adquiridos en torno de los temas expuestos durante el curso, pues de ellos dependen que egresen con una formación más sólida, así como atender la actualización de docentes en cuanto al uso de plataformas educativas.

Por último, el papel de las y los docentes es crucial para crear las estrategias necesarias lo más equitativas posibles durante la pandemia, enfocadas a una mejor comunicación y aprendizaje (guiando y fomentando la autonomía del aprendizaje). Aunque poco podemos incidir en el desempeño estudiantil, cuando los factores sociales, el contexto de origen del estudiante y su disposición no lo permiten.

\section{REFERENCIAS}

Blanco, E., Solís P. y Robles H. (2014) Caminos desiguales. Trayectorias educativas y laborales de los jóvenes de la Ciudad de México. COLMEX- INEE. 
Bourdieu, P. y Passeron J.C. (2003). Los herederos. Los estudiantes y la cultura. Siglo XXI.

De Garay, A., Miller, D. y Montoya, I. (2016). Una misma institución, estudiantes diferentes. Los universitarios de nuevo ingreso de las unidades Azcapotzalco y Cuajimalpa de la UAM. Revista Sociológica, 31(88), 95-140. https://www.redalyc.org/ pdf/3050/305045555004.pdf

Giddens, A (1995). Introducción, Elementos de la teoría de la estructuración, Estructura, sistema, reproducción social, Teoría de la estructuración, investigación empírica y crítica social, en Giddens, La constitución de la sociedad: Bases para la teoría de la estructuración, pp. 15-33.

González, L. (2020). Estrés académico en estudiantes universitarios asociados a la pandemia por COVID-19. ESPACIO 1+D, Innovación más desarrollo, 9(25). http:// www.dgei.unam.mx/hwp/wpcontent/uploads/2021/05/07e13951aa7762ed8a2f8b3885927f23.pdf

Guzmán, C. (2013). Introducción, en Los estudiantes y la universidad. Integración, experiencias e identidades (pp. 7 -23). ANUIES

Hernández, R., Fernández, C. y Baptista, M. (1997). Metodología de la investigación. McGraw Hill.

INEGI (2018). Encuesta Nacional de la Dinámica Demográfica (ENADID). Base de datos. SNIEG, Información de Interés Nacional. https:/ / www.inegi.org.mx/programas/ enadid/2018/

INEGI (2020, 10 de agosto). Comunicado de prensa núm. 393/20. Estadísticas a propósito del día internacional de la juventud. https:/ / www.inegi.org. $\mathrm{mx} /$ contenidos/saladeprensa/aproposito/2019/Juventud2019 Nal.pdf

INEGI (2021). Encuesta para la Medición del Impacto COVID-19 en la Educación ECOVID-ED. https:/ / www.inegi.org.mx/contenidos/investigacion/ecovided/2020/doc/ecovid ed 2020 presentacion resultados.pdf

Marinoni, G., Van't Land, H. \& Jensen, T. (2020). The Impact of COVID-19 on Higher Education Around the World IAU Global Survey Report. Retrieved from https:/ / www.iauaiu.net/IMG/pdf/iau covid19 and he survey report final may 2020.pdf

MEJOREDU (2020). Experiencias de las comunidades educativas durante la contingencia sanitaria por COVID-19. Educación media superior. Cuadernos de investigación educativa. Comisión Nacional para la Mejora Continua de la Educación. México. https: / / www. mejoredu.gob.mx/publicaciones/informe-de-resultados/experiencias-de-las-comunidades-educativas-durante-la-contingencia-sanitaria-por-covid-19-eb

Miguel, J. (2020). La educación superior en tiempos de pandemia: una visión desde dentro del proceso formativo. Revista Latinoamericana de Estudios Educativos, 50 (ESPECIAL), 13-40. https:// doi.org/10.48102/rlee.2020.50.ESPECIAL.95

Navarrete, Z., Manzanilla, H. y Ocaña, L. (2020) Políticas implementadas por el gobierno mexicano frente al COVID-19. El caso de la educación básica. Revista Latinoamericana de Estudios Educativos, 50 (ESPECIAL), 143-172. https:// www.redalyc.org/ journal $/ 270 / 27063237025 / \mathrm{html} /$ 
Navia, A., Czarny, G. y Salinas, S. (2021). Estudiar fuera de la universidad en pandemia: estudiantes indígenas en México. Revista Ciencia y Cultura, 25(46), 69-87. http:/ / www. scielo.org.bo/scielo.php?script=sci arttext\&pid=S2077-33232021000100004\&ln$\mathrm{g}=\mathrm{es} \& \operatorname{tlng}=\mathrm{es}$

Ordorika, I. (2020). Pandemia y educación superior. Revista de la educación superior, 49(194), 1-8. https: / / doi.org/10.36857/ resu.2020.194.1120

Organización Internacional del Trabajo (2020). Los jóvenes y la COVID-19: efectos en los empleos, la educación, los derechos y el bienestar mental. Informe de la Encuesta 2020. https://www.ilo.org/wcmsp5/groups/public/ed_emp/documents/publication/wcms 753054.pdf

Ramírez, R. (2013) Cambiar, interrumpir o abandonar. La construcción de experiencias de los estudiantes en su tránsito por una institución de educación superior tecnológica. ANUIES. 2da. edición.

Rieble, S. y Viteri, A. (2020). COVID-19. ¿Estamos preparados para el aprendizaje en línea? CIMA América Latina y el Caribe. Nota 20. Banco Interamericano para el Desarrollo. https://publications.iadb.org/es/publications/spanish/document/Nota-CIMA--20-COVID-19-Estamos-preparados-para-el-aprendizaje-en-linea.pdf

Silva, M. y Rodríguez, A. (2013). ¿Cómo viven su primer año universitario los jóvenes provenientes de sectores de pobreza? En Carlota Guzmán Gómez (Coord.). Los estudiantes y la universidad: integración, experiencias e identidades. ANUIES. 